Rocem Pol Jiménez López

\title{
Desenvolvimento de Atuadores Tridimensionais Baseados em Músculos Artificiais Dielétricos de Uma ou Múltiplas Camadas
}

Dissertação de Mestrado

Dissertação apresentada como requisito parcial para obtenção do título de Mestre pelo Programa de Pós-Graduação em Engenharia Mecânica da PUC-Rio.

Orientador: Marco Antonio Meggiolaro 


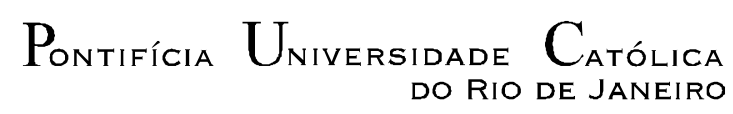

Rocem Pol Jiménez López

\title{
Desenvolvimento de Atuadores Tridimensionais Baseados em Músculos Artificiais Dielétricos de uma ou Múltiplas Camadas
}

\begin{abstract}
Dissertação apresentada como requisito parcial para obtenção do grau de Mestre pelo Programa de PósGraduação em Engenharia Mecânica do Centro Técnico Científico da PUC-Rio. Aprovada pela Comissão Examinadora abaixo assinada.
\end{abstract}

Prof. Marco Antonio Meggiolaro

Orientador

Departamento de Engenharia Mecânica - PUC-Rio

Prof. Mauro Speranza Neto

Departamento de Engenharia Mecânica - PUC-Rio

Prof. Luciano Luporini Menegaldo Instituto Militar de Engenharia

Prof. José Eugenio Leal Coordenador Setorial do Centro Técnico Científico - PUC-Rio 
Todos os direitos reservados. É proibida a reprodução total ou parcial do trabalho sem autorização da universidade, do autor e do orientador.

\section{Rocem Pol Jiménez López}

Graduou-se em Engenharia Mecatrônica Universidad Nacional de Ingeniería Lima Perú em 2006.

Ficha Catalográfica

Jiménez López, Rocem Pol

Desenvolvimento de atuadores tridimensionais baseados em músculos artificiais dielétricos de uma ou múltiplas camadas / Rocem Pol Jiménez López ; orientador: Marco Antonio Meggiolaro. 2009.

121 f. : il. (color.) ; $30 \mathrm{~cm}$

Dissertação (Mestrado em Engenharia Mecânica)-Pontifícia Universidade Católica do Rio de Janeiro, Rio de Janeiro, 2009.

Inclui bibliografia

1. Engenharia mecânica - Teses. 2. Músculo artificial. 3. Atuadores binários. 4. Comportamento viscoelástico. $5 . \quad$ Visão estéreo. I. Meggiolaro, Marco Antonio. II. Pontifícia Universidade Católica do Rio de Janeiro. Departamento de Engenharia Mecânica. III. Título. 
A minha mãe María, meu pai Diómodes, minha irmã Magali, meu irmão Leboni, minha avó Dionisia, meu cunhado Julio, minha sobrinha Luana e meu sobrinho 


\section{Agradecimentos}

Ao Professor Marco Antonio Meggiolaro pela orientação e paciência durante o desenvolvimento do curso de mestrado.

Ao CAPES, FAPERJ e à PUC-Rio, pelos auxílios concedidos, sem os quais este trabalho não poderia ter sido realizado.

Ao Departamento de Engenharia Mecânica da PUC-Rio, pelo ensino e colaboração comigo.

A todas aquelas pessoas que contribuíram no desenvolvimento da dissertação. 


\section{Resumo}

López, Rocem Pol Jiménez; Meggiolaro, Marco Antonio. Desenvolvimento de Atuadores Tridimensionais baseados em Músculos Artificiais Dielétricos de Uma ou Múltiplas Camadas. Rio de Janeiro, 2009. 121p. Dissertação de Mestrado - Departamento de Engenharia Mecânica, Pontifícia Universidade Católica do Rio de Janeiro.

Músculos artificiais são versáteis para o projeto de atuadores pois, assim como os músculos naturais, podem ter pequenas dimensões ou serem agrupados para alcançar maiores dimensões. A capacidade do músculo de permitir a construção de atuadores sem partes móveis permite uma grande economia de energia, evitando atritos por deslizamento, menor desgaste, e baixos ruídos.Músculos artificiais são usados para desenvolver atuadores contínuos ou binários, utilizando diversos tipos de configurações para aproveitar ao máximo as vantagens que oferecem estes materiais. Músculos artificiais eletrostrictivos, acionados por altas tensões, já estão sendo utilizados em aplicações comerciais. Circuitos eletrônicos estão sendo projetados para trabalhar com altas tensões elétricas e interagir com estes tipos de músculos. Diversos tipos de material estão sendo avaliados para a implementação destes músculos.Este trabalho visa desenvolver um atuador de configuração cilíndrica de acionamento elétrico, por efeito capacitivo. $\mathrm{O}$ atuador é acionado por 3 músculos artificiais em configuração paralela, construídos a partir do elastômero acrílico VHB4905, o qual possui características visco-elásticas e baixo custo de produção. São analisados distintos modelos constitutivos dos músculos, baseados em molas e amortecedores em série e em paralelo.São desenvolvidos métodos de calibragem para calcular os parâmetros dos modelos matemáticos dos músculos a partir de dados obtidos em laboratório. Um método de medição baseado em processamento de imagens e teoria de visão estéreo, desenvolvido especificamente para este trabalho, permite que a posição da extremidade do atuador seja medida de forma não-invasiva, sem interferir em seu movimento, e sem a necessidade de colocar sensores ou instrumentos de medição. Os resultados mostram que os modelos matemáticos são eficientes para descrever o comportamento do atuador.

\section{Palavras-chave}

Músculo Artificial, Atuadores binários, Comportamento Viscoelástico, Visão Estéreo. 


\section{Abstract}

López, Rocem Pol Jiménez; Meggiolaro, Marco Antonio (Advisor). Development of Three-Dimensional Actuators Based on Dielectric Artificial Muscles of One or Multiple Layers. Rio de Janeiro, 2009. 121p. MSc Dissertation - Departamento de Engenharia Mecânica, Pontifícia Universidade Católica do Rio de Janeiro.

Artificial muscles are versatile for the actuator projects, because, as well as the natural muscles, they can have small dimensions or they are grouped to obtain big dimensions. Muscle capacity to allow construction of actuators without movable parts allows great economy of energy, avoiding attritions for sliding, lessening waste, and reducing noise.Artificial muscles are used to develop continuous or binary actuators, using different types of configurations to take advantage to the benefit that offer these materials. Eletrostrictive artificial muscles, worked by high tensions, are already being used in commercial applications. Electronic circuits are being projected to work with high electric tensions and to interact with these types of muscles. Many types of materials are being evaluated for the implementation of these muscles.This work looks for to develop a cylindrical configuration actuator of electric activation, for capacitive effect. The actuator is activated by 3 artificial muscles in parallel configuration, built starting from the acrylic elastomer VHB4905, which possesses viscouselastic characteristics and low production cost. Different models of the muscles are analyzed, based on springs and shock absorbers in series and parallel.Calibrate methods are developed to calculate parameters of the mathematical models of the muscles starting from data obtained at laboratory. Measurement method based on processing of images and theory of stereo vision, specifically developed for this work, allows no-invasive measurement of the actuator's extremity, without interfering in actuator's movement, and without the need to put sensor or measurement instruments. The results show that mathematical models are efficient to describe the behavior of the actuator.

\section{Keywords}

Artificial muscles, Binary actuators, viscous-elastic behavior, Stereo Vision. 


\section{Sumário}

1 INTRODUÇÃO 20

1.1. Motivação 21

1.2. Objetivos do Trabalho 22

1.3. Trabalhos anteriores 23

1.4. Descrição do Trabalho 25

2 MODELAGEM DO ATUADOR 26

2.1. Concepção do Atuador 26

2.2. Modelagem Matemática do Atuador 31

2.3. Modelagem Matemática do Músculo Artificial 34

2.4. Modelagem Matemática da Mola Central 39

2.5. Equações Dinâmicas do atuador 41

3 SOLUÇÃO NUMÉRICA 43

3.1. Matriz Jacobiana do Sistema de Equações 43

3.2. Solução Segundo o Newton-Raphson 47

3.3. Solução Segundo o Newton-Raphson para Kelvin-Voigt + A 48

4 MEDIÇÃO DA POSIÇÃO USANDO VISÃO ESTÉREO 50

4.1. Obtenção do Par Estéreo 51

4.2. Obtenção Automática dos Pontos Correspondentes 57

4.3. Cálculo das Matrizes de Projeção das Câmeras 61

4.4. Cálculo dos Pontos em Três Dimensões 63

4.5. Cálculo da Posição e Orientação da Base Superior 66

5 SIMULACAO, EXPERIMENTOS E CALIBRAÇÃO DO ATUADOR 69

5.1. Simulação do Atuador 69

5.2. Sistema Experimental 70

5.3. Resultados da medição estéreo 75 
5.4. Calibração do Atuador

5.5. Resultados da calibração

5.6. Validação

6 CONCLUSÕES

Referências bibliográficas

Apêndice A - Construção do Atuador

Apêndice B - Calculo dos Parâmetros do Atuador para K- V

Apêndice C - Relações entre Rotação e a Velocidade Angular

Apêndice D - Relações entre os Ângulos e Velocidade

Apêndice E - Derivadas com vetores

Apêndice F - Ajuste por mínimos quadrados de um plano 


\section{Lista de figuras}

Figura 1: Elastômero dielétrico VHB4905. 20

Figura 2: Evolução dos robôs (fonte: NIAC, EUA). 21

Figura 3: Atuador binário (fonte: NIAC, EUA). 22

Figura 4: Aplicação dos músculos artificiais em pequenos robôs. 22

Figura 5: (a) BRAID III. (b) BRAID IV 23

Figura 6: Atuador modelado por Bystronski. 23

Figura 7: Modelo matemático do atuador. 24

Figura 8: Teste de moldura fixa com VHB 4910 sem tensão. 24

Figura 9: Modulo elevador de tensão. 25

Figura 10: (a) Objetivo. (b) Mola central. 26

Figura 11: (a) Forma geral do atuador. (b) Vista de corte. 27

Figura 12: (a) Atuador triangular. (b) Atuador elíptico. 27

Figura 13: (a) Esquema do atuador. (b) Projeto do atuador. 28

Figura 14: Atuador cortado por dois planos. 28

Figura 15: (a) Base inferior. (b) Base inferior, fios e músculo. 29

Figura 16: (a) Anéis fixadores inferiores. (b) Base e anéis. 29

Figura 17: (a) Camadas concêntricas. (b) Projeto do Atuador pronto. $\quad 30$

Figura 18 (a) Movimento translacional. (b) A Base translada e gira. 31

Figura 19: (a) Esquema do músculo. (b) Sub-divisão do músculo. 32

Figura 20: (a) Molas e amortecedores. (b) Posição de um elemento. 32

Figura 21: (a) Translação. (b) Rot. em X. (c) Rot. em $Y$. (d) Rot. em Z. 33

Figura 22: (a) Posições dos elementos. (b) Mola-amortecedor. 34

Figura 23: Modelo de Kelvin-Voigt. 35

Figura 24: Modelo Zener. $\quad 36$

Figura 25: Modelo Kelvin-Voigt + Amortecedor. 36

Figura 26: Modelo de Burgers. $\quad 37$

Figura 27: (a) Mola. (b) Mola depois de ser transladada e girada. $\quad 39$

Figura 28: Diagrama vetores e força. $\quad 40$

Figura 29: (a) Forças do atuador. (b) Diagrama de corpo livre. 41 
Figura 30: (a) Objeto da medição. (b) Câmeras para medir. 51

Figura 31: (a) Interseção de duas retas. (b) Grupo de pontos. 52

Figura 32: (a) Erros na reconstrução 3D. (b) Erros menores. 52

Figura 33: (a) Mesma distância representada por números diferentes de pixels. (b) Mesma distância de pontos quadro a quadro representada por números diferentes de pixels.

Figura 34: (a) Três pontos para definir a posição e orientação do objeto. (b) Muitos pontos para obter informação com melhor precisão. 54

Figura 35: Objeto côncavo tipo bloco em duas vistas. 54

Figura 36: (a) Posição das câmeras para suas respectivas

quinas. (b) Posição das câmeras nos experimentos.

Figura 37: (a) Vista segundo direção $-i-j+k$. (b) Vista

segundo direção $-i-\sqrt{2} j+k$.

56

Figura 38: (a) Imagem capturada pela primeira câmera

("câmera da direita"). (b) Imagem capturada pela segunda câmera ("câmera da esquerda").

56

Figura 39: (a) Objeto com dois sistemas de coordenadas para a calibração de cada câmera. (b) Imagem da quina direita capturada pela câmera da direita com sistema de coordenas na sua quina superior esquerda.

Figura 40: (a) Pontos na Imagem que ajudam na busca

de pontos. (b) Imagem do objeto isolado.

Figura 41: (a) Magnitude do gradiente da imagem.

(b) Direção do gradiente da imagem. 58

Figura 42: Três grupos calculados segundo k-means. 59

Figura 43: Seis grupos de retas.

Figura 44: (a) Mostra os seis grupos de retas juntos e os quatro pontos de referência recalculados. (b) Os pontos de referência recalculados com maior precisão.

Figura 45: (a) Quadro atual mostrando a vizinhança onde se pegará uma pequena imagem para a correlação cruzada.

(b) Quadro novo com a vizinhança onde é feita a busca 
do novo ponto.

Figura 46: (a) Pontos de referência no quadro atual. (b) Pontos de referência no quadro novo e de onde eles vieram.

Figura 47: Par estéreo com a correspondência de dois pares de pontos 61

Figura 48: Pontos correspondentes do objeto e da imagem dele. 62

Figura 49: (a) Objeto colocado na base superior para calibrar as câmeras. (b) Posição arbitrária do objeto depois de que a base superior foi liberada.

Figura 50: Construção do Ponto em três dimensões.

Figura 51: Par estéreo, a reconstrução em três dimensões e a correspondência de um dos pontos.

Figura 52: (a) Sistema de coordenadas no mundo real na base inferior e o sistema de coordenadas da base superior.

(b) Vetores unitários associados aos eixos da base superior.

Figura 53 (a) O vetor unitário é a interseção de dois planos ajustados segundo seus respectivos pontos. (b) Projeção dos pontos a um plano normal ao vetor unitário.

Figura 54: (a) Ajuste de duas retas com a condição de serem perpendiculares. (b) Centro de sistema de coordenadas a seis elementos de distância da média de dois pontos.

Figura 55: Simulação do atuador de duas camadas.

Figura 56: Par de quadros capturados pelo par de webcams.

Figura 57: Imagem real na parte esquerda e Imagem da simulação na parte direita. 70

Figura 58: Partes do elevador de tensão elétrica.

Figura 59: Atuador. $\quad 71$

Figura 60: Par estéreo e o emissor de luz 72

Figura 61: Câmera webcam para obter o vídeo do atuador. 72

Figura 62: Componentes do experimento excluindo os computadores. 73

Figura 63: (a) Propriedades da carga. (b) Se coloca a massa e o objeto de medição ao atuador de uma camada.

Figura 64: (a) Captura das imagens do par estéreo. 
(b) Captura de imagem do atuador.

Figura 65: Atuador quebrado ao final dos experimentos. 74

Figura 66: Partes importantes para análises dos resultados. 75

Figura 67: Giros e posições no experimento um. 76

Figura 68: Giros e posições no experimento dois. 77

Figura 69: Giros e posições no experimento três. 77

Figura 70: Giros e posições no experimento quatro. 78

Figura 71: Giros e posições no experimento cinco. 78

Figura 72: Giros e posições no experimento seis. 78

Figura 73: Giros e posições no experimento sete. 79

Figura 74: Giros e posições no experimento oito. $\quad 79$

Figura 75: Giros e posições no experimento nove. $\quad 80$

Figura 76: Giros e posições no experimento dez. 80

Figura 77: Giros e posições no experimento onze. 81

Figura 78: (a) Oz nos experimentos um, quatro, sete e dez.

(b) $\gamma$ nos dez primeiros experimentos. 81

Figura 79: Posições e rotações são medidas a longo do tempo 83

Figura 80: Sub-índice $j$ para as partes sem tensão elétrica, $k$ para as partes com tensão elétrica e $i$ para todo o conjunto. 83

Figura 81: Modelo matemático KV. 84

Figura 82: Parâmetros calculados nos dez experimentos. $\quad 87$

Figura 83: Ajuste para o modelo KV. 88

Figura 84: Resultados do experimento oito para a validação. 88

Figura 85: Resultados do experimento onze para a validação. $\quad 89$

Figura 86: Medidas da base inferior em escala 1:1 com duas

vistas em detalhe em escala 4:1 e duas seções para cada detalhe também em escala 4:1.

Figura 87: Medidas da base superior em escala 1:1 com uma vista em detalhe em escala 4:1 e uma seção para o detalhe também em escala 4:1. 97

Figura 88: Medidas do anel fixador pequeno inferior. 98

Figura 89: Medidas do anel fixador pequeno superior em escala 1:1 98

Figura 90: Medidas do anel fixador maior inferior. 99 
Figura 91: medidas do fixador maior superior em escala 1:1. 100

Figura 92: As seis peças de garolite. 100

Figura 93: Anéis Fixadores separados com ajuda de parafusos. 101

Figura 94: (a) Fixadores e músculos. (b) Fios condutores. 101

Figura 95: (a) Superfície. (b) Músculo colado aos fixadores. 101

Figura 96: (a) Músculo esticado. (b) Músculo com capacitores. 102

Figura 97: (a) Camadas coladas. (b) Atuador construído. 102

Figura 98: Atuador de uma camada ao final dos experimentos. 103

Figura 99: Sub-índices. 104

Figura 100: Modelo matemático KV. 105 


\section{Lista de tabelas}

Tabela 1: Tipos de modelos para cada elemento. 35

Tabela 2: Modelos matemáticos para cada elemento do músculo. 38

Tabela 3: Resumo das vantagens do objeto. 55

Tabela 4: Resumo de experimentos: 82 


\section{Lista de símbolos}

$\alpha$ : Giro no eixo $X$.

$\beta$ : Giro no eixo $Y$.

$\gamma$ : Giro no eixo $Z$.

$\theta$ : Ângulo entre o eixo $X$ e a posição final da partição número $i$.

$\Delta \theta:$ Ângulo formado entre duas partições consecutivas.

$A$ : Matriz de rotação da base superior.

$A_{\alpha}:$ Matriz de rotação gerada pelo giro $\alpha$.

$A_{\beta}$ : Matriz de rotação gerada pelo giro $\beta$.

$A_{\gamma}$ : Matriz de rotação gerada pelo giro $\gamma$.

$\hat{a}$ : Vetor unitário na direção $Z$.

$\hat{a}_{z}$ : Componente do vetor $\hat{a}$ no eixo $Z o$.

$\hat{a}_{z}$ : Ângulo formado pelo vetor $\hat{a}$ e o eixo $Z o$.

$B_{i}$ : Vetor que indica a direção da partição número $i$ do músculo.

$\hat{b}_{i}$ : vetor unitário de $B_{i}$.

$\left\|B_{i}\right\|:$ Modulo de $B_{i}$.

$c_{A_{i}}$ : Primeira constante de amortecimento do modelo KV+A na partição $i$.

$c_{A_{2}}$ : Segunda constante de amortecimento do modelo KV+A na partição $i$.

$C_{B_{1}}$ : Primeira constante de amortecimento do modelo Burgers na partição $i$.

$C_{B_{2}}$ : Segunda constante de amortecimento do modelo Burgers na partição $i$.

$C B K_{F}$ :Variável auxiliar para o calculo.

$C B K_{T}$ :Variável auxiliar para o calculo.

$C D K_{F}$ :Variável auxiliar para o calculo.

$C D K_{T}$ :Variável auxiliar para o calculo

$C E I_{T}$ :Variável auxiliar para o calculo. 
$C g$ : Centro de massa da carga.

$c_{K V_{i}}$ : Constante de amortecimento do modelo KV para à partição número $i$.

${ }_{C K V} V_{i}$ : Constante de amortecimento do modelo Zener na partição número $i$.

$C T_{F}$ :Variável auxiliar para o calculo.

$C T_{T}$ :Variável auxiliar para o calculo.

$C V I_{F}$ :Variável auxiliar para o calculo.

$C V I_{T}$ :Variável auxiliar para o calculo.

$C V K_{F}$ :Variável auxiliar para o calculo..

$C V K_{T}$ :Variável auxiliar para o calculo.

$c z_{i}$ : Constante de amortecimento do modelo Zener na partição número $i$.

$d$ : Posição do $G g$ referente ao sistema de coordenadas da base superior.

$d A$ : Derivada parcial de $A$ referente a quaisquer das seis variáveis.

$D_{i}$ : Vetor que inicia em $G g$ e termina em $P f_{i}$.

$d B_{i}$ : Derivada parcial de $B_{i}$ referente a quaisquer das seis variáveis.

$d\left\|B_{i}\right\|:$ Derivada parcial de $\left\|B_{i}\right\|$ referente a quaisquer das seis variáveis.

$d \hat{b}_{i}$ : Derivada parcial de $\hat{b}_{i}$ referente a quaisquer das seis variáveis.

$d F$ : Derivada parcial de $F$ referente a quaisquer das seis variáveis.

$d f_{i}$ : Derivada parcial de $f_{i}$ referente a quaisquer das seis variáveis.

$d \varepsilon_{i}$ : Derivada parcial de $\varepsilon_{i}$ referente a quaisquer das seis variáveis.

$d \dot{\varepsilon}_{i}$ : Derivada parcial de $\dot{\varepsilon}_{i}$ referente a quaisquer das seis variáveis.

$d \ddot{\varepsilon}_{i}$ : Derivada parcial de $\ddot{\varepsilon}_{i}$ referente a quaisquer das seis variáveis.

dFmol : Derivada parcial de Fmol referente a quaisquer das seis variáveis.

$d h_{i}$ : Derivada parcial de $h_{i}$ referente a quaisquer das seis variáveis.

$d \dot{h}_{i}$ : Derivada parcial de $\dot{h}_{i}$ referente a quaisquer das seis variáveis.

$d \ddot{h}_{i}$ : Derivada parcial de $\ddot{h}_{i}$ referente a quaisquer das seis variáveis.

$d P f_{i}$ : Derivada parcial de $P f_{i}$ referente a quaisquer das seis variáveis.

$d O$ : Derivada parcial de $O$ referente a quaisquer das seis variáveis.

$d T i$ : Derivada parcial de $T i$ referente a quaisquer das seis variáveis. 
dTmol : Derivada parcial de Tmol referente a quaisquer das seis variáveis.

$d \omega_{i}$ : Derivada parcial de $\omega_{i}$ referente a quaisquer das seis variáveis.

$d \dot{\omega}_{i}$ : Derivada parcial de $\dot{\omega}_{i}$ referente a quaisquer das seis variáveis.

$\varepsilon_{i}$ : Deformação da partição número $i$.

$F:$ Sistema de equações de forcas e torques.

$f_{i}$ : Força vetorial na partição número $i$.

Fg : Força de gravidade.

Fmol : Força que a mola exerce à base superior.

$H$ : Vetor que contem os três vetores de giro $\alpha, \beta$ e $\gamma$.

$h_{i}$ : Força escalar na partição número $i$.

$I$ :Matriz de inércia da carga.

$\hat{i}$ : Vetor unitária no eixo $X$.

$\hat{j}$ Vetor unitária no eixo $Y$.

$J F$ : Matriz Jacobiana de $F$.

$K$ : Constante de rigidez da mola central.

$\hat{k}$ : Vetor unitário no eixo $Y$.

$k_{A_{i}}$ : Constante de rigidez do modelo $\mathrm{KV}+\mathrm{A}$ para a partição número $i$.

$k_{B_{i}}$ : Primeira constante de rigidez do modelo Burgers na partição número $i$.

$k_{B_{2}}$ : Segunda constante de rigidez do modelo Burgers na partição número $i$.

$k_{i}$ : Constante de rigidez equivalente no equilibro estático.

$k_{K V_{i}}$ : Constante de rigidez do modelo KV para a partição número $i$.

$\hat{k} o$ : Vetor unitário na direção Zo .

$k z_{1}$ : Primeira constante de rigidez do modelo Zener na partição número $i$.

$k z_{2}$ : Segunda constante de rigidez do modelo Zener na partição número $i$.

$L$ : Comprimento natural da mola central.

$l_{i}$ : Longitude natural da partição número $i$.

$m$ : Massa da carga.

$M K V_{S V}:$ Variável auxiliar para o calculo.

$M K V_{C V}$ :Variável auxiliar para o calculo. 
$N_{K_{1}}$ : Variável auxiliar para o calculo.

$N_{K_{2}}$ :Variável auxiliar para o calculo.

$N_{K_{3}}$ :Variável auxiliar para o calculo.

$O:$ Centro da base superior.

$O x$ : Primeira componente de $O$.

Oy: segunda componente de $O$.

$O z$ : quarta componente de $O$.

$\|O\|:$ Modulo de $O$.

$\hat{o}:$ Vetor unitário de $O$.

$\hat{o}_{n}$ : Vetor unitário normal ao plano definido pelo eixo Zo e o vetor $\hat{o}$.

$\hat{o}_{t}$ : Vetor unitário que pertence ao plano definido pelo eixo Zo e o vetor $\hat{o}$.

$\rho c_{o}$ : Constante de amortecimento por unidade de comprimento.

$P f_{i}$ : Ponto final da partição número $i$ do músculo.

$\rho k_{o}$ : Constante de rigidez por unidade de comprimento.

$P o_{i}$ : Ponto inicial da partição número $i$ do músculo.

Rbase : Raio das bases.

$R F$ :Variável auxiliar para o calculo.

$R K V_{S V}$ :Variável auxiliar para o calculo.

$R K V_{C V}$ :Variável auxiliar para o calculo.

$R R F:$ Variável auxiliar para o calculo.

$R R T$ :Variável auxiliar para o calculo.

$R T$ :Variável auxiliar para o calculo.

Tmol: Torque, referente ao centro de massa da carga, que exerce Fmol à carga.

$T i$ : Torque, referente ao centro de massa da carga, que exerce $f_{i}$ à carga.

$\Delta t:$ Diferencial de tempo.

$\mu_{i}$ : Numero de coluna numa imagem.

$v_{i}$ : Numero de fila numa imagem.

$\omega$ : Velocidade angular.

$[\omega]$ : $\omega$ ordenado em uma matriz para o produto vetorial.

$W$ : Matriz que relaciona $\omega$ e $\dot{H}$. 